Mathematical Research Letters 4, 473-479 (1997)

\title{
THE SPECTRUM OF THE LAPLACIAN ON A MANIFOLD OF NONNEGATIVE RICCI CURVATURE
}

\author{
JIAPING WANG
}

\section{$\S 1$ Introduction}

The study of the spectrum of the Laplacian on a complete noncompact Riemannian manifold has received much attention during the past decade or so. In particular, it has been conjectured and partially verified that the spectrum of the Laplacian acting on the space of $L^{2}$ functions (the $L^{2}$ spectrum) is the half line $[0, \infty)$ when the underlying manifold has nonnegative Ricci curvature. J. F. Escobar and A. Freire $([\mathrm{E}],[\mathrm{E}-\mathrm{F}])$ have dealt with the case that the manifold has nonnegative sectional curvature and proved among other things that this is true provided that the exponential map from the normal bundle of a soul of the manifold is a diffeomorphism onto the manifold. Later, J. Li in [Li] showed that this is also true for a Ricci nonnegative manifold possessing a pole, namely, the exponential map at some point is a diffeomorphism from the tangent space onto the manifold. Recently, the author was informed that H. Donnelly has considered the Ricci nonnegative manifolds with maximal volume growth. Among other things, he established also that the $L^{2}$ spectrum of the Laplacian is $[0, \infty)$ in this case. This last result has also been obtained independently by $\mathrm{N}$. Castañeda in $[\mathrm{C}]$. In this short note, our purpose is to demonstrate the validity of the conjecture without imposing extra assumptions on the manifold. In fact, we shall prove more generally that the $L^{2}$ spectrum of the Laplacian on a complete noncompact Riemannian manifold with asymptotically nonnegative Ricci curvature is given by the half line $[0, \infty)$. Recall that a complete manifold $\left(M^{n}, g\right)$ has asymptotically nonnegative Ricci curvature if there exists a small constant $\delta(n)>0$ depending only on $n$ such that for some point $q \in M$, the Ricci curvature satisfies $\operatorname{Ric}_{M}(x) \geq-\delta(n) r^{-2}(x)$, where $r(x)$, the distance from $x$ to $q$, is sufficiently large. Note that here we do not address the problem whether there is any eigenvalue for the Laplacian.

\section{$\S 2$ Proof of the Theorem}

In this section, we shall give the proof of the result that the $L^{2}$ spectrum of the Laplacian on a complete noncompact Riemannian manifold with asymptotically nonnegative Ricci curvature is given by the half line $[0, \infty)$. The argument

Received April 9, 1997.

The author was supported by an AMS Centennial Fellowship.

This work was done when the author was visiting MIT. 
relies on a result in $[\mathrm{S}]$ which is stated below and utilizes the testing functions constructed in $[\mathrm{E}]$ and $[\mathrm{Li}]$. We first recall that the Laplacian $\Delta$ of a complete manifold $(M, g)$ is defined on $C_{c}^{\infty}(M)$, the space of smooth functions with compact support on $M$, and can be uniquely extended into a self-adjoint operator on the space $L^{2}(M)$, which is denoted by $\Delta_{2}$. It is well-known then that the semigroup $\exp \left(t \Delta_{2}\right)$ extends to a strongly continuous contraction semigroup on any $L^{p}(M), p \in[1, \infty)$. Its generator is denoted by $\Delta_{p}$. For $p=\infty, \Delta_{p}$ is defined to be the adjoint of $\Delta_{1}$, i.e., $\Delta_{\infty}=\Delta_{1}^{*}$. It is clear that $C_{c}^{\infty}(M)$ is contained in the domain of $\Delta_{p}$ and $\Delta_{p}=\Delta$ on $C_{c}^{\infty}(M)$ for all $p \in[1, \infty]$. The volume of the manifold $(M, g)$ is said to grow uniformly subexponentially if for any $\epsilon>0$, there exists a constant $C<\infty$ such that $V_{x}(r) \leq C e^{\epsilon r} V_{x}(1)$ for all $x \in M$ and $r \geq 1$, where $V_{x}(r)$ is the volume of the geodesic ball $B_{x}(r)$ in $M$. The following result is proved in $[\mathrm{S}]$.

Theorem 1. [S] If the Ricci curvature of a complete noncompact manifold $(M, g)$ is bounded from below and the volume of $(M, g)$ grows uniformly subexponentially, then the spectrum of $\Delta_{p}$ acting on $L^{p}(M)$ is independent of $p \in[1, \infty]$.

It has been verified in $[\mathrm{S}]$ that the volume of manifold $(M, g)$ grows uniformly subexponentially if its Ricci curvature is bounded from below by a function $K(x)$ on $M$ satisfying $\liminf \sin _{x \rightarrow \infty} K(x) \geq 0$. In particular, this is true for a manifold with asymptotically nonnegtaive Ricci curvature. Before we proceed to prove our main result, we collect some facts into the following lemma.

Lemma 2. Let $M^{n}$ be a complete noncompact Riemannian manifold of dimension $n$ such that its Ricci curvature satisfies Ric $\operatorname{Ri}_{M}(x) \geq-\delta(n) r^{-2}(x)$, where $r(x)$ is the distance from $x$ to $q$, on $M \backslash B_{q}(a)$ and $\delta(n)>0$ depending only on $n$ is a small constant, and has lower bound $-(n-1) K$ on $B_{q}(a)$, where $K \geq 0$. Then

(1) $\Delta r(x) \leq \frac{c_{1}(n, a, K)}{r}$ for some constant $c_{1}$.

(2) there exist constants $c_{2}(n, a, K)>1$ and $c_{3}(n, a, K)>0$ such that for all $r>0$ and $1 \leq \beta \leq 2, V_{q}(2 r) \leq c_{2} V_{q}(r)$ and $A_{q}(\beta r) \leq c_{3} A_{q}(r)$, where $V_{q}(r)$ is the volume of the geodesic ball $B_{q}(r)$ and $A_{q}(r)$ the area of the geodesic sphere $\partial B_{q}(r)$.

(3) there exists $\epsilon(n, a, K)>0$ such that for all $r>0$ sufficiently large, $V_{q}(\epsilon r) \leq \frac{1}{2}\left(\frac{1}{c_{2}}\right)^{2} V_{q}(r)$ and $A_{q}(\epsilon r) \leq \frac{c_{4}(n, a, K)}{r}\left[V_{q}(2 r)-V_{q}(\epsilon r)\right]$ for some positive constant $c_{4}$.

Proof. Part (1) is the standard Laplacian comparison theorem. We refer to the book [S-T] for a proof. Part (2) follows from the well-known volume comparison argument. The details have been given in $[\mathrm{L}]$ and $[\mathrm{L}-\mathrm{T}]$. We prove part $(3)$ by using the argument in $[\mathrm{C}-\mathrm{G}-\mathrm{T}]$ and $[\mathrm{L}-\mathrm{S}]$. In fact, we shall prove more generally that there exists a constant $c(n)>0$ such that for $a \leq r \leq R$,

$$
V_{q}(r) \leq c \frac{r}{R} V_{q}(R)
$$


Fix a constant $\beta>\frac{2}{2^{1 / n}-1}$. For $x \in M$ with $r(x)=T=\left(2 \sum_{j=0}^{k} \beta^{j}-1-\beta^{k}\right) r$, let $\gamma$ be a minimal geodesic joining $q$ and $x$ with $\gamma(0)=q$ and $\gamma(T)=x$. Define $x_{i}=\gamma\left(t_{i}\right)$, where $t_{0}=0$ and $t_{i}=\left(2 \sum_{j=0}^{i} \beta^{j}-1-\beta^{i}\right) r, i=1,2, \ldots, k$. Let $R_{i}=\beta^{i} r$ for $i=0,1, \ldots, k$. Then the relative volume comparison theorem (see [C-G-T] or $[\mathrm{L}]$ ) implies that

$$
V_{x_{i}}\left(R_{i}\right) \geq T_{i}\left(V_{x_{i}}\left(R_{i}+2 R_{i-1}\right)-V_{x_{i}}\left(R_{i}\right)\right) \geq T_{i} V_{x_{i-1}}\left(R_{i-1}\right)
$$

where

$$
T_{i}=\frac{\int_{0}^{R_{i}} \sinh ^{n-1} \sqrt{k\left(x_{i}, R_{i}+2 R_{i-1}\right)} t d t}{\int_{R_{i}}^{R_{i}+2 R_{i-1}} \sinh ^{n-1} \sqrt{k\left(x_{i}, R_{i}+2 R_{i-1}\right)} t d t},
$$

and in general $-(n-1) k(x, t)$ denotes the lower bound of the Ricci curvature on $B_{x}(t)$. The assumption on $R i c_{M}$ yields

$$
\sqrt{k\left(x_{i}, R_{i}+2 R_{i-1}\right)} \leq \delta^{1 / 2}(n)\left(\sum_{j=0}^{i-2} \beta^{j}-1\right)^{-1} r^{-1}
$$

for sufficiently large $i$. Since $\beta>1$ is fixed, we conclude that there is a fixed constant $c(n)$ such that

$$
\left(R_{i}+2 R_{i-1}\right) \sqrt{k\left(x_{i}, R_{i}+2 R_{i-1}\right)} \leq c(n) \delta^{1 / 2}(n),
$$

which can be made arbitrarily small by the smallness assumption on $\delta(n)$. Hence

$$
\begin{aligned}
T_{i} & =\frac{\int_{0}^{R_{i} \sqrt{k\left(x_{i}, R_{i}+2 R_{i-1}\right)}} \sinh ^{n-1} t d t}{\int_{R_{i} \sqrt{k\left(x_{i}, R_{i}+2 R_{i-1}\right)}}^{\left(R_{i}+2 R_{i-1}\right) \sqrt{k\left(x_{i}, R_{i}+2 R_{i-1}\right)}} \sinh ^{n-1} t d t} \\
& \approx \frac{R_{i}^{n}}{\left(R_{i}+2 R_{i-1}\right)^{n}-R_{i}^{n}} \\
& =\frac{\beta^{n}}{(\beta+2)^{n}-\beta^{n}}
\end{aligned}
$$

by simply approximating sinh $t$ with $t$. Therefore,

$$
V_{x_{k}}\left(R_{k}\right) \geq V_{q}(r) \Pi_{i=1}^{k} T_{i} \geq c\left(\frac{\beta^{n}}{(\beta+2)^{n}-\beta^{n}}\right)^{k} V_{q}(r) .
$$

Hence,

$$
\begin{aligned}
V_{q}(2 T) & \geq V_{x_{k}}\left(R_{k}\right) \\
& \geq c\left(\frac{\beta^{n}}{(\beta+2)^{n}-\beta^{n}}\right)^{k} V_{q}(r) \\
& \geq c \frac{2 T}{r} V_{q}(r) .
\end{aligned}
$$


Since $k$ is arbitrary, we conclude that

$$
V_{q}(R) \geq c \frac{R}{r} V_{q}(r)
$$

for all $R \geq r \geq a$.

Finally, to show the last inequality, we note that from (2), there exists a constant $c_{5}(n, a, K)$ such that

$$
c_{5} \epsilon r A_{q}(\epsilon r) \leq \int_{\frac{1}{2} \epsilon r}^{\epsilon r} A_{q}(t) d t \leq V_{q}(\epsilon r) .
$$

So we have

$$
A_{q}(\epsilon r) \leq \frac{c_{6}}{r} V_{q}(\epsilon r)
$$

However,

$$
V_{q}(2 r)-V_{q}(\epsilon r) \geq V_{q}(r)-V_{q}(\epsilon r) \geq\left(2 c_{2}^{2}-1\right) V_{q}(\epsilon r) .
$$

Therefore, we conclude that

$$
A_{q}(\epsilon r) \leq \frac{c_{4}}{r}\left[V_{q}(2 r)-V_{q}(\epsilon r)\right]
$$

for some constant $c_{4}$ depending on $n, a$ and $K$. The proof is completed.

Now we are ready to prove our main result. In the following, we shall denote by $c$ a generic constant which may depending on $n, a$ and $K$ but not on $k$.

Theorem 3. Let $M$ be a complete noncompact Riemannian manifold. Suppose that the Ricci curvature of $M$ is asymptotiaclly nonnegative. Then the spectrum of the Laplacian $\Delta_{p}$ acting on the space $L^{p}(M)$ is $[0, \infty)$ for all $p \in[1, \infty]$.

Proof. Since $M$ has asymptotiaclly nonnegative Ricci curvature, Theorem 1 is applicable and implies that the spectrum of $\Delta_{p}$ is independent of $p \in[1, \infty]$. So we need only to show that the spectrum of $\Delta_{1}$ is $[0, \infty)$. We may fix a point $q \in$ $M$ and assume that the Ricci curvature of $M$ satisfies $\operatorname{Ric}_{M}(x) \geq-\delta(n) r^{-2}(x)$, where $r(x)$ is the distance from $x$ to $q$, on $M \backslash B_{q}(a)$ and $\delta(n)>0$ depending only on $n$ is a small constant, and has a lower bound $-(n-1) K$ on the ball $B_{q}(a)$, where $a$ and $K$ are nonnegative constants, and $n$ is the dimension of the manifold. Note that $r(x)$ is a Lipschitz function with $|\nabla r(x)|=1$ almost everywhere. Also, by Lemma $2, \Delta r(x) \leq \frac{c}{r(x)}$ in the sense of distribution. Let $E$ be the cut-locus of the point $q \in M$ and $\Omega=M \backslash E$. Since the volume of the set $E$ is zero, by the Fubini's theorem, the area of the set $\partial B_{q}(t) \cap E$ is zero for almost all $t$. We call such $t$ to be permissible. By mollifying the function $r(x)$, one obtains a sequence of smooth functions $\left\{f_{k}\right\}$ on $M$ such that $f_{k}$ converges to $r(x)$ uniformly on compact subsets of $M$, and $\left|\nabla f_{k}\right| \leq 2$ on $M$ 
and $\left|\nabla f_{k}-\nabla r\right| \leq \frac{1}{k}$ on $\Omega$, and $\Delta f_{k}(x) \leq \frac{c}{r(x)}+\frac{1}{k}$ on $M$. We note that in particular $\frac{\partial f_{k}}{\partial r} \geq 0$ on $\partial B_{q}(t) \cap \Omega$ for all $t>0$ and if $t$ is permissible, then

$$
0 \leq \int_{\partial B_{q}(t)} \frac{\partial f_{k}}{\partial r}=\int_{\partial B_{q}(t) \cap \Omega} \frac{\partial f_{k}}{\partial r} \leq 2 A_{q}(t)
$$

We now follow $[\mathrm{E}]$ and $[\mathrm{Li}]$ and define for any $\lambda \geq 0$ a sequence of functions

$$
\phi_{k}(x)=\eta_{k} \psi\left(\frac{f_{k}(x)}{k}\right) e^{i \sqrt{\lambda} f_{k}(x)}
$$

where $\eta_{k}=\left[V_{q}(2(k+1))-V_{q}(\epsilon(k-1))\right]^{-1}$, and $\psi(r)$ is a smooth function such that $\psi(r)=1$ for $2 \epsilon \leq r \leq 1$ and $\psi(r)=0$ for $r \geq 2$ or $r \leq \epsilon$ with $0 \leq \psi(r) \leq 1$, $\left|\psi^{\prime \prime}(r)\right| \leq C(\epsilon)$. Here and in the following, $\epsilon>0$ is a fixed constant chosen to satisfy Lemma 2. It is clear that $\phi_{k}(x)$ is a smooth function with compact support on $M$. Also, we may assume without loss of generality that $2(k+1)$ and $\epsilon(k-1)$ are permissible. A direct computation gives

$$
\begin{aligned}
(\Delta+\lambda) \phi_{k}(x)= & \eta_{k} \frac{1}{k^{2}} \psi^{\prime \prime}\left(\frac{f_{k}}{k}\right) e^{i \sqrt{\lambda} f_{k}}\left|\nabla f_{k}\right|^{2}+2 i \sqrt{\lambda} \eta_{k} \frac{1}{k} \psi^{\prime}\left(\frac{f_{k}}{k}\right) e^{i \sqrt{\lambda} f_{k}}\left|\nabla f_{k}\right|^{2} \\
& +\eta_{k} \frac{1}{k} \psi^{\prime}\left(\frac{f_{k}}{k}\right) e^{i \sqrt{\lambda} f_{k}} \Delta f_{k}+i \sqrt{\lambda} \eta_{k} \psi\left(\frac{f_{k}}{k}\right) e^{i \sqrt{\lambda} f_{k}} \Delta f_{k} \\
& +\lambda\left(1-\left|\nabla f_{k}\right|^{2}\right) \phi_{k}
\end{aligned}
$$

Thus, we conclude that

$$
\begin{aligned}
\left|(\Delta+\lambda) \phi_{k}(x)\right| \leq & \frac{4}{k^{2}} \eta_{k}\left|\psi^{\prime \prime}\left(\frac{f_{k}}{k}\right)\right|+\frac{8 \sqrt{\lambda}}{k} \eta_{k}\left|\psi^{\prime}\left(\frac{f_{k}}{k}\right)\right| \\
& +\eta_{k} \frac{1}{k}\left|\psi^{\prime}\left(\frac{f_{k}}{k}\right)\right|\left|\Delta f_{k}\right|+\sqrt{\lambda} \eta_{k}\left|\psi\left(\frac{f_{k}}{k}\right)\right|\left|\Delta f_{k}\right| \\
& +\left.\lambda \eta_{k}|1-| \nabla f_{k}\right|^{2} \mid \psi\left(\frac{f_{k}}{k}\right) .
\end{aligned}
$$

Since

$$
\Delta f_{k}(x) \leq \frac{c_{1}(n, a, K)}{r(x)}+\frac{1}{k},
$$

if we let $\left(\Delta f_{k}\right)^{+}$be the positive part of $\Delta f_{k}$, then

$$
\left(\Delta f_{k}\right)^{+} \leq \frac{c_{1}(n, a, K)}{r}+\frac{1}{k} .
$$

Note that

$$
\left|\Delta f_{k}\right|=2\left(\Delta f_{k}\right)^{+}-\Delta f_{k}
$$


From (3) and using (1), (4), (5), we obtain that for $k$ sufficiently large

$$
\begin{aligned}
& \int_{M}\left|(\Delta+\lambda) \phi_{k}(x)\right| d x \leq \frac{4 \eta_{k}}{k^{2}} \int_{M}\left|\psi^{\prime \prime}\left(\frac{f_{k}}{k}\right)\right| d x+\frac{8 \sqrt{\lambda}}{k} \eta_{k} \int_{M}\left|\psi^{\prime}\left(\frac{f_{k}}{k}\right)\right| d x \\
& +\eta_{k} \frac{1}{k} \int_{M}\left|\psi^{\prime}\left(\frac{f_{k}}{k}\right)\right|\left|\Delta f_{k}\right| d x+\sqrt{\lambda} \eta_{k} \int_{M}\left|\psi\left(\frac{f_{k}}{k}\right)\right|\left|\Delta f_{k}\right| d x \\
& +\left.\lambda \eta_{k} \int_{M}|1-| \nabla f_{k}\right|^{2} \mid \psi\left(\frac{f_{k}}{k}\right) \\
& \leq \frac{c}{k} \eta_{k} \int_{\epsilon(k-1) \leq r(x) \leq 2(k+1)} d x+c \eta_{k} \int_{\epsilon(k-1) \leq r(x) \leq 2(k+1)}\left|\Delta f_{k}(x)\right| d x \\
& \leq \frac{c}{k}+c \eta_{k} \int_{\epsilon(k-1) \leq r(x) \leq 2(k+1)}\left[2\left(\Delta f_{k}\right)^{+}-\Delta f_{k}\right] d x \quad \Delta f_{k}(x) d x \\
& \left.\leq \frac{c}{k}+c \eta_{k} \int_{\epsilon k-1 \leq r(x) \leq 2 k+1} \frac{2 c_{1}}{r(x)} d x-c \eta_{k} \int_{\epsilon(k-1) \leq r(x) \leq 2(k+1)} \frac{\partial f_{k}}{\partial r}-\int_{\partial B_{q}(2(k+1))} \frac{\partial f_{k}}{\partial r}\right] \\
& \leq \frac{c}{k}+c \eta_{k}\left[\int_{\partial B_{q}(\epsilon(k-1))}\right. \\
& \leq \frac{c}{k}+c \eta_{k} A_{q}(\epsilon(k-1)),
\end{aligned}
$$

where we have used the Green's formula. By Lemma 2, $\eta_{k} A_{q}(\epsilon(k-1)) \leq \frac{c}{k}$. Therefore, we conclude from (6) that for $k$ sufficiently large

$$
\int_{M}\left|(\Delta+\lambda) \phi_{k}(x)\right| d x \leq \frac{c}{k}
$$

Hence

$$
\lim _{k \rightarrow \infty} \int_{M}\left|(\Delta+\lambda) \phi_{k}(x)\right| d x=0 .
$$

On the other hand, using the definition of $\phi_{k}(x)$ and Lemma 2, we get for $k$ sufficiently large

$$
\begin{aligned}
\int_{M}\left|\phi_{k}(x)\right| d x & \geq \frac{V_{q}(k-1)-V_{q}(2 \epsilon(k+1))}{V_{q}(2(k+1))-V_{q}(\epsilon(k-1))} \\
& \geq \frac{V_{q}(k-1)}{V_{q}(2(k+1))}-\frac{V_{q}(2 \epsilon(k+1))}{V_{q}(2(k+1))} \\
& \geq \frac{V_{q}(k-1)}{V_{q}(4(k-1))}-\frac{1}{2}\left(\frac{1}{c_{2}}\right)^{2} \\
& \geq\left(\frac{1}{c_{2}}\right)^{2}-\frac{1}{2}\left(\frac{1}{c_{2}}\right)^{2} \\
& \geq \frac{1}{2}\left(\frac{1}{c_{2}}\right)^{2} .
\end{aligned}
$$

From (8) and (9), one concludes that $\lambda$ is in the spectrum of $\Delta_{1}$. Since $\lambda \geq 0$ is arbitrary, we conclude that the spectrum of $\Delta_{1}$ is $[0, \infty)$. The same assertion then also holds for the spectrum of $\Delta_{p}$ for $p \in[1, \infty]$. The theorem is proved. 


\section{Acknowledgements}

I would like to express my gratitude to Professor Gang Tian and the Mathematics Department of MIT for their hospitality. I would also like to thank Professor Peter Li for his interest in this work.

\section{References}

[C] N. Castañeda, Spectrum of the Laplacian on manifolds of nonnegative Ricci curvature and maximal volume growth, preprint.

[C-G-T] J. Cheeger, M. Gromov, and M. Taylor, Finite propagation speed, kernel estimates for functions of the Laplace operator, and the geometry of complete Riemannian manifolds, J. Diff. Geom. 17 (1982), 15-53.

[E] J. F. Escobar, On the spectrum of the Laplacian on complete Riemannian manifolds, Comm. Partial Diff. Eq. 11 (1986), 63-85.

[E-F] J. F. Escobar and A. Freire, The spectrum of the Laplacian of manifolds of positive curvature, Duke Math. J. 65 (1992), 1-21.

[L] P. Li, Lectures notes on geometric analysis, Lecture Notes Ser., 6, Research Institute of Math., Seoul National Univ., 1993.

[L-S] P. Li and R. Schoen, $L^{p}$ and mean value properties of subharmonic functions on Riemannian manifolds, Acta Math. 153 (1984), 279-301.

[L-T] P. Li and L. F. Tam, Green's functions, harmonic functions, and volume comparison, J. Diff. Geom. 41 (1995), 277-318.

[Li] J. Li, Spectrum of the Laplacian on a complete Riemannian manifold with nonnegative Ricci curvature which possess a pole, J. Math. Soc. Japan 46 (1994), 213-216.

[S] K. Sturm, On the $L^{p}$-spectrum of uniformly elliptic operators on Riemannian manifolds, J. Funct. Anal. 118 (1993), 442-453.

[S-T] R. Schoen and S. T. Yau, Lectures on differential geometry, Conf. Proc. Lecture Notes Geom. Topology, International Press, Cambridge, 1994.

Department of Mathematics, Massachusetts Institute of Technology, Cambridge, MA 02139-4307

E-mail address: jwang@math.mit.edu 